\title{
Solid lipid nanoparticles with enteric coating for improving stability, palatability, and oral bioavailability of enrofloxacin
}

This article was published in the following Dove Medical Press journal: International Journal of Nanomedicine

\author{
Chao $\mathrm{Li}^{\prime}$ \\ Kaixiang Zhou' \\ Dongmei Chen ${ }^{1,2}$ \\ Wei Xu' \\ Yanfei Tao' \\ Yuanhu Pan ${ }^{2}$ \\ Kuiyu Meng ${ }^{2}$ \\ Muhammad Abu Bakr \\ Shabbir ${ }^{2}$ \\ Qianying Liu' \\ Lingli Huang ${ }^{2}$ \\ Shuyu Xie' \\ 'National Reference Laboratory of \\ Veterinary Drug Residues (HZAU) \\ and MAO Key Laboratory for \\ Detection of Veterinary Drug \\ Residues, Wuhan Hubei, China; ${ }^{2}$ MOA \\ Laboratory for Risk Assessment \\ of Quality and Safety of Livestock \\ and Poultry Products, Huazhong \\ Agricultural University, Wuhan \\ 430070, Wuhan Hubei, China
}

Background: The poor palatability, variable oral bioavailability, stimulation to gastric mucosa, and light instability limited the application of enrofloxacin (ENR). The enteric granules combining solid lipid nanoparticles (SLNs) with enteric coating were explored to overcome these disadvantages.

Materials and methods: ENR-loaded SLNs were produced by a hot homogenization and ultrasonic emulsification method and the enteric granules with SLNs as inner core were prepared by wet granulation followed by coating using polyacrylic resin II (PRII). The formulation was optimized by using orthogonal or single factor test screening.

Results: The optimal SLNs with loading capacity (LC) and price as inspection indexes were consisted of $10 \mathrm{~mL} \mathrm{3 \%}$ polyvinyl alcohol per $0.8 \mathrm{~g}$ ENR and $2.4 \mathrm{~g}$ octadecanoic acid. The sizes, LC, polydispersion index, and zeta potential of the SLNs were $308.5 \pm 6.3 \mathrm{~nm}, 15.73 \% \pm 0.31 \%$, $0.352 \pm 0.015$, and $-22.3 \mathrm{mv}$, respectively. The best enteric granules were used $15 \% \mathrm{PRII}$ as coating materials. The release of the enteric granules in simulated intestine fluid (SIF, $\mathrm{pH}=8$ ) was significantly faster than in simulated gastric fluid ( $\mathrm{SGF}, \mathrm{pH}=2)$ and simultaneously slower than those of SLNs and native ENR. The granules showed good stability in influencing factor experiment. The granules displayed a similar daily feed intake as the control group and higher daily feed intake than ENR powder and single-coating granules. Compared to the ENR soluble powder, the area under the plasma concentration-time curve and mean retention time of the enteric granules after intragastric administration were increased from $4.26 \pm 0.85 \mu \mathrm{g} \mathrm{h} / \mathrm{mL}$ and $6.80 \pm 2.28$ hours to $11.24 \pm 3.33 \mu \mathrm{g} \mathrm{h} / \mathrm{mL}$ and $17.97 \pm 4.01$ hours, respectively.

Conclusion: The enteric granules combination SLNs with enteric coating significantly improved the stability, palatability, sustained-release performance and oral bioavailability of ENR. The novel technology will be a potential measure to overcome the similar disadvantages of other drugs.

Keywords: enrofloxacin, solid lipid nanoparticles, enteric coating, palatability, bioavailability, light stability

\section{Introduction}

Enrofloxacin (ENR), known as ethyl ciprofloxacin, is the first animal-specific fluoroquinolone and successfully developed by Bayer AG of Germany. ${ }^{1}$ It has been widely used in veterinary clinic because of its broad antibacterial spectrum, strong bactericidal activity, low adverse reactions, and high postantibacterial effect. ${ }^{2}$ However, ENR has a bitter taste. ${ }^{3,4}$ The poor palatability limits its oral administration since the animals with keen sense tastes, especially for pigs and cows, exhibit more possibility to refuse the bitter drugs. ${ }^{5}$ Oral formulations currently available in market cannot mask the bitter taste
National Reference Laboratory of Veterinary Drug Residues (HZAU) and MAO Key Laboratory for Detection of Veterinary Drug Residues, No.I Shizishan, Wuhan Hubei 430070, China Tel +86278728 73238221

Fax +862787672232

Email snxsyl@|26.com 
of ENR and thus limit the oral clinical applications for pigs. In addition, the drug has short elimination half-life $\left(T_{1 / 2 \beta}\right)$ and mean residence time (MRT) in most mammals and variable bioavailability due to its poor aqueous solubility. Therefore, the control-release formulation with good palatability, little gastric irritation, and improved absorption is necessary.

Some taste-masking formulations were prepared to improve the palatability of ENR. Chun et al prepared a ENR carbopol complex to mask the bitter taste, while its taste-masking effect is uncertain due to lack of direct animal experiment. ${ }^{4}$ The double-coated ENR microparticles might effectively mask the bitter of ENR but the release data of microparticles in simulated gastric fluid (SGF) were not provided. ${ }^{3}$ The formulations prepared by hydrogel materials (sodium alginate, chitosan) had a low $\mathrm{pH}$-dependent release. ${ }^{6,7}$ So the bulk release of these hydrogel preparations in SGF might result in stomach irritation due to the irritant effect ofenrofloxacin on the gastric mucosa. This might influence animal oral compliance. The reduction of ENR release in stomach might be a potential way to achieve excellent release since ENR has a maximum solubility in low $\mathrm{pH}(>100 \mathrm{mg} / \mathrm{mL}$ in $\mathrm{pH}=1) .{ }^{8}$ EC Besides, ENR has high photolysis. ${ }^{9}$ It should be stored away from light and thus increases the storage and transportation costs. Therefore, the development of a new dosage with good palatability, low stimulation to stomach and good stability is urgent for ENR.

Solid lipid nanoparticles (SLNs) are a novel nanosized drug delivery system using high-melting natural or synthetic solid lipids as backbone materials because of their good physiological compatibility and outstanding physicochemical property (nanosize and large specific surface area effect). ${ }^{10}$ It is often used to improve the permeability and palatability, ${ }^{11}$ achieve controlling release, ${ }^{12,13}$ and enhance oral bioavailability. ${ }^{14}$ However, the release of ENR from SLNs in stomach is very rapid due to the maximum solubility of ENR in gastric juice. In addition, the SLNs could not completely entrap the drug. Therefore, mere nanoparticles may not effectively decrease the release of ENR in stomach and achieve the best sustained release and entirely mask the bitter taste.

Coating technology including enteric coating is a production process in which macromolecule material is wrapped on drugs by chemical or physical method. ${ }^{15}$ After coating, the contact chance between the drug and the taste buds of animal is reduced, ${ }^{16}$ and the drug could be isolated from direct exposure of the natural light. The coating of functional materials (ie, water-insoluble, enteric-soluble materials) can impart specific target release properties of drug in the gastrointestinal tract. In recent years, coating techniques have been widely used to increase the palatability and delivery of drugs to specific target sites. Choi and Kim coated the peonja using Eudragit acrylic resin, which well masked the bitterness of the drug. ${ }^{17}$ It was also reported that the taste of gabapentin was significantly improved when coated with the mixture of gelatin, partially hydrogenated soybean oil, and glyceryl monostearate. ${ }^{18}$ Alsulays et al reported that the cumulative release of lansoprazole enteric coated was $<10 \%$ in the acidic condition, while up to $80 \%$ in the alkaline buffer stage. ${ }^{19}$ The stability of all-trans-retinoic acid by fluid-bed coating under high illumination $(4,500 \pm 500 \mathrm{~lx})$ was reported to be enhanced. ${ }^{20}$ However, the simple coating might not remarkably improve the palatability because of the greatest bitterness of ENR. It happens that coating tablets or granules with enteric materials might reduce the dissolution of drug in stomach but not obtain sufficient taste masking.

In order to overcome the disadvantages of insufficient taste masking and controlled release performance of simple coating and SLNs, ENR taste-masking enteric granules were creatively prepared by combining SLNs with coating in this study. The formulation was optimized by using orthogonal test or single factor screening. The properties, stability, palatability, and the sustained release in vitro and in vivo were further evaluated.

\section{Materials and methods Materials}

ENR standard (98\% ENR content) was bought from Dr Ehrenstorfer. ENR ( $\geq 96.0 \%$ ENR content) was purchased from Wuhan Konglong Century Technology Development Co., Ltd. (Wuhan, China). ENR soluble powder was purchased from Guangdong Wens Dahuanong Biotechnology Co., Ltd. (Guangdong, China). Octadecanoic acid and docosanoic acid were obtained from Aladdin (Shanghai, China). Polyvinyl alcohol (PVA) and poloxamer 188 were bought from Sigma (St. Louis, MO, USA). Polyvinyl pyrrolidone $\left(\mathrm{PVP}_{\mathrm{K} 30}\right)$ was obtained from Sinopharm Group Chemical Reagent Co., Ltd. (Shanghai, China). Polyacryl resin II (PRII) was purchased from Shanghai Source Biological Technology Co., Ltd (Shanghai, China). Ethyl cellulose (EC) and sodium carboxymethyl cellulose were purchased from Sinopharm Group Chemical Reagent Co., Ltd.

\section{Animals}

Twenty-seven clinically healthy three-way hybrid pigs (15-20 kg) were obtained from Huazhong Agricultural University (HAZU) Livestock Engineering Center (Wuhan, China). The pigs were acclimatized at laboratory animal 


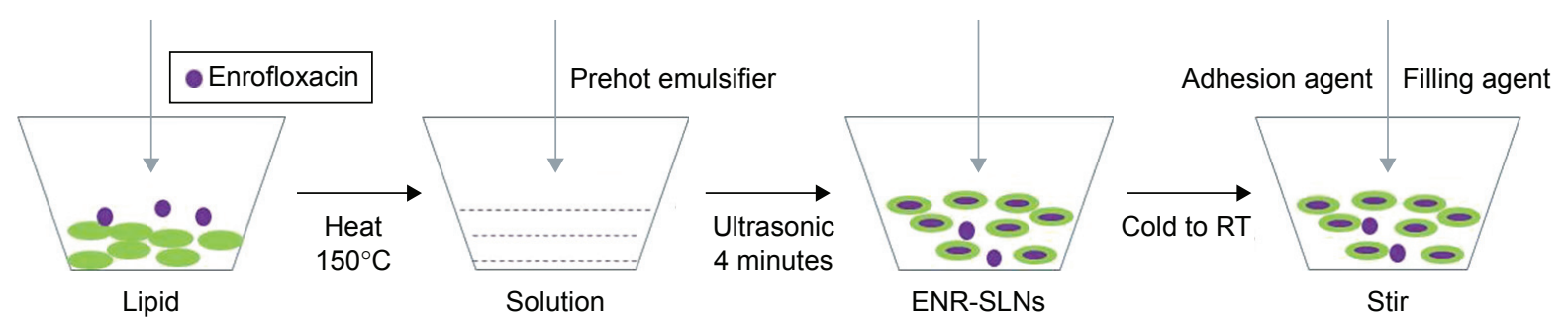

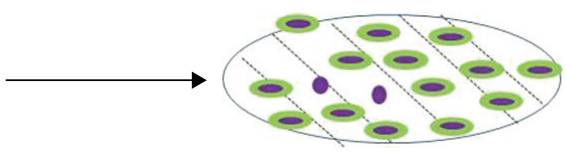

Primary soft material

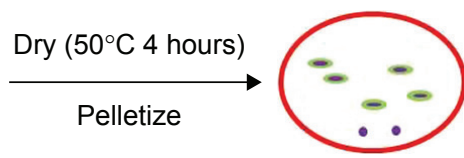

Pelletize

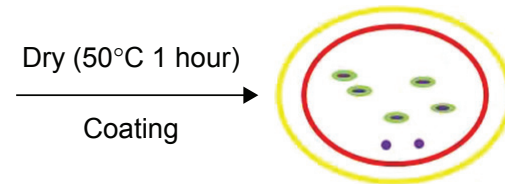

Enteric granules

Figure I The production process of enrofloxacin enteric granules containing SLNs inner core.

Abbreviations: ENR-SLNs, enrofloxacin-loaded SLNs; RT, room temperature; SLNs, solid lipid nanoparticles.

rooms at the National Reference Laboratory of Veterinary Drug Residues (HZAU) and were freely fed on drug-free feed and made to drink for 1 week. The relative humidity and temperature of the breeding environment were maintained at $45 \%-65 \%$ and $18^{\circ} \mathrm{C}-25^{\circ} \mathrm{C}$, respectively. All the experimental protocols were authorized by the Institutional Animal Care and Use Committee at HAZU (approval number: HZAUSW-2018-009) and followed the guidelines of Hubei Science and Technology Agency.

\section{Preparation of ENR enteric granules}

The production process flow of the ENR enteric granules was shown in Figure 1.

\section{Preparation of ENR-loaded SLNs}

The SLNs were produced by a hot melt homogenization with ultrasonic emulsification method. Briefly, a certain amount of ENR was dissolved in the melted solid lipid under stirring by a magnetic stirrer (9001, Shanghai Huxi Analysis Instrument Factory Co., Ltd., Shanghai, China). After complete dissolution, a certain volume and concentration of preheated emulsifier solution was swiftly poured into the above melted lipid solution under stirring to form a primary emulsion. The primary emulsion was sonicated using $6 \mathrm{~mm}$ microprobes with 95\% amplitude (VCX 130 Vibra-CellTM, Sonics \& Materials, Inc., Newtown, CT, USA) for 4 minutes to form a hot $\mathrm{O} / \mathrm{W}$ emulsion. In the preparation process of $\mathrm{O} / \mathrm{W}$ emulsion, the emulsifier was optimized by orthogonal experiment using drug loading capacity (LC) of SLNs as assessment index. The type, concentration, and volume of the emulsifiers were also selected as variables. The corresponding levels are PVA, PVP, poloxamer 188; 1\%, 2\%, 3\%; $10 \mathrm{~mL}, 15 \mathrm{~mL}$, $20 \mathrm{~mL}$, respectively. After determination of the variables and their levels, the design of the orthogonal test was used SPSS software (version 20, IBM) (Table 1). In the orthogonal test, the composition of one to nine SLNs was $15 \mathrm{~mL} 3 \%$

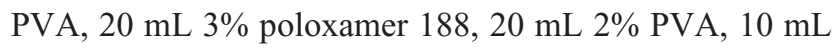
1\% PVA, $15 \mathrm{~mL} \mathrm{1 \%} \mathrm{poloxamer} \mathrm{188,} 20 \mathrm{~mL} \mathrm{1 \%} \mathrm{PVP} \mathrm{K30,}$ $10 \mathrm{~mL} \mathrm{3 \%}$ PVP K30, $15 \mathrm{~mL}$ 2\% PVP K30, and $10 \mathrm{~mL} \mathrm{2 \%}$ poloxamer 188 per $0.8 \mathrm{~g}$ ENR and $2.4 \mathrm{~g}$ octadecanoic acid, respectively. The hot $\mathrm{O} / \mathrm{W}$ nanoemulsion was cooled down under the room temperature to form SLNs.

\section{Preparation of coating granules using SLNs as inner core}

After formulation of the ENR-loaded SLNs (ENR-SLNs), a certain amount of sucrose and starch was, respectively, added into the nanosuspension during stirring to prepare preliminary soft material. The preliminary soft material was placed in a dry oven (DGX-9243B-1, Fuma Test Equipment CO., Ltd., Shanghai, China) $\left(50^{\circ} \mathrm{C}, 4\right.$ hours $)$ to prepare the suitable soft material for pelletizing. After pelletized by using granulator (YK-60, Zhongcheng Pharmacy Machine Co., Ltd., Changsha, China), the prepared granules were dried again $\left(50^{\circ} \mathrm{C}, 1\right.$ hour $)$ to remove the water. The dry granules were coated with $10 \%(\mathrm{~W} / \mathrm{W})$ ethyl cellulose or $5 \%, 10 \%$, and $15 \%(\mathrm{~W} / \mathrm{W})$ polyacrylic resin (PRII) using coating pan (BY-400, Zhongcheng Pharmacy Machine Co., Ltd.).

Table I Factors and levels of the L9 $\left(3^{4}\right)$ orthogonal design

\begin{tabular}{l|l|l|l}
\hline \multirow{2}{*}{ Variables } & \multicolumn{3}{|l}{ Level } \\
\cline { 2 - 4 } & $\mathbf{I}$ & $\mathbf{2}$ & $\mathbf{3}$ \\
\hline Type & PVA & $\mathrm{PVP}_{\mathrm{K} 30}$ & Poloxamer I88 \\
Concentration & $1 \%$ & $2 \%$ & $3 \%$ \\
Volume $(\mathrm{mL})$ & 10 & 15 & 20 \\
\hline
\end{tabular}

Abbreviations: PVA, polyvinyl alcohol; $\mathrm{PVP}_{\mathrm{K} 30}$, polyvinyl pyrrolidone K30. 
After coating, the ENR taste-masking enteric granules were obtained. The optimal content of PRII for coating was evaluated by in vitro release performance.

\section{Preparation of simple coating enteric granules}

The preparation process of simple coating enteric granules was the same as the above ENR double taste-masking enteric granules except the process of production of ENR-SLNs. Briefly, $60 \%$ starch, $15 \%$ sucrose, and 10\% ENR were completely mixed. Then, $5 \%$ starch using as an adhesive agent was produced into starch paste with $50 \mathrm{~mL}$ water by boiling method. The prepared starch paste was mixed with the above mixture and formed granules via pelletizing. The granules were coated by $10 \%$ PRII ( $750 \mathrm{~mL} 2 \%$ alcohol solution).

\section{Characterization of ENR-SLNs}

Determination of particle size, polydispersion index (PDI), and zeta potential

The morphology of SLNs was observed by scanning electron microscope (JSM-6390LV, NTC, Co., Ltd., Tokyo, Japan). The size, PDI, and zeta potential were measured by using Zetasizer ZX3600 (Malvern Instruments, Worcestershire, UK) and laser particle size analyzer BT-9300S (Better, Liaoning, China) at $25^{\circ} \mathrm{C}$. The samples were diluted in distilled water to make sure that the concentration was $2.7 \mathrm{mg} / \mathrm{mL}$ for the tests of size and PDI, and $0.3 \mathrm{mg} / \mathrm{mL}$ for the measurement of zeta potential to get the optimum kilo counts per second of 20-400. All determinations were repeated in triplicate by using independent preparations.

\section{Loading capacity (LC) and encapsulation efficiency (EE) of SLNs}

The determination of LC and EE of SLNs was described in our previous work. ${ }^{21}$ Briefly, the nanosuspensions were collected by centrifugation at 14,000 rpm (Hitachi Centrifugation CR21 GIII; Hitachi Koki Co., Ltd., Japan) for 60 minutes at $4^{\circ} \mathrm{C}$. The free ENR in the supernatant was measured by Waters 2695 series HPLC equipped with Waters 2587 UV detector (Waters Corp., Milford, MA, USA) to determine the EE. The precipitated SLNs were resuspended in distilled water and lyophilized for 48 hours (Freeze Dry System; Labconco, Missouri USA) for determination of LC. After freeze drying, $10 \mathrm{mg}$ dried nanoparticles were added in a $15 \mathrm{~mL}$ tube containing $10 \mathrm{~mL}$ acetonitrile/water solution (V/V; $1: 1)$ and put in a boiling water bath to destroy the nanoparticles. The SLNs after heating was added to the volume of $10 \mathrm{~mL}$ and centrifuged at $8,000 \mathrm{rpm}$ for 10 minutes. The supernatant after filtration was injected into HPLC for analysis. The assay was repeated in triplicate using different samples from in dependent preparations. The EE and LC were defined as follows:

$$
\begin{gathered}
\text { Weight of ENR added } \\
\mathrm{EE}(\%)=\frac{- \text { Weight of ENR in supernatant }}{\text { Weight of ENR added }} \times 100 \\
\mathrm{LC}(\%)=\frac{\text { Weight of ENR in SLNs }}{\text { Weight of dried SLNs }} \times 100
\end{gathered}
$$

\section{In vitro release}

In vitro release of the ENR granules was measured in SGF ( $\mathrm{pH}=2,100 \mathrm{~mL}$ contained $2.0 \mathrm{~g} \mathrm{NaCl}$ and $3.2 \mathrm{~g}$ pepsin and then $\mathrm{HCl}$ was used to adjust the $\mathrm{pH}$ to 2 ) and simulated intestine fluid (SIF; $\mathrm{pH}=8,1,000 \mathrm{~mL}$ SIF contained $6.8 \mathrm{~g}$ $\mathrm{KH}_{2} \mathrm{PO}_{3}$ and $10 \mathrm{~g}$ trypsin and then $\mathrm{NaOH}$ solution was used to adjust the $\mathrm{pH}$ to 8 ) of pigs by using Dissolution tester RC806 (Tianjing Tiandatianfa Co., Ltd. Tianjing, China). The maximum solubility of ENR in SGF and SIF was 1.99 and $0.31 \mathrm{mg} / \mathrm{mL}$, respectively. According to the sink condition, $1 \mathrm{~g}$ enteric granules (contained $100 \mathrm{mg}$ ENR) or 155 mg enteric granules (contained $15.5 \mathrm{mg}$ ENR) $(\mathrm{n}=3)$ were placed in a dissolution cup containing $200 \mathrm{~mL}$ buffer at $38^{\circ} \mathrm{C}$ under rotating propeller stirring at $100 \mathrm{rpm}$. One milliliter of samples was periodically collected from the dissolution cup at fixed times to measure the released drug, and the same volume of fresh SGF or SIF was added after each sampling to keep a constant volume. The drug concentration in the release media was determined by HPLC. The cumulative release curve was plotted using the cumulative release rate and time as ordinate and abscissa. In vitro release of the ENR granules with different kinds of and content of coating materials were done to select the optimum formulation. The in vitro release of native ENR, ENR-SLNs, and simple coating granules were used as control using the same process. The granularity and drying loss of all the kinds of granules meet the requirement of Chinese Veterinary Pharmacopoeia (2015). ${ }^{22}$

\section{Palatability experiment}

The palatability of ENR enteric granules was studied by pig feeding and water intake experiment. Briefly, 15 pigs were randomly divided into five groups with each group consisting of 3 pigs. The five groups included blank group, ENR powder group, simple enteric-coated granules, ENR enteric granules with $10 \%$ sucrose, and ENR enteric granules with $15 \%$ sucrose groups. Before the experiment, the average daily feed intake of each group was determined for continuous 
3 days to ensure that the selected pigs were healthy, had normal appetite and excluded the individual possible errors. In the experiment period, pigs were free to feed on medicated feed with different ENR formulations for continuous 5 days. At 9 am every day, the enough feed without or mixed ENR formulations was added into the trough, and the remainder feed was weighted at 9 am of the next day. The daily feed intake of each group was equal to the added feed of the first day minus the remaining feed of the next day. After 7 days cleaning periods of ending of the daily feed intake experiment, 6 pigs were randomly selected to study the palat of ENR-SLNs via the daily water intake for 5 days. Briefly, six pigs were randomly divided into control group and ENRSLNs group $(n=3)$. At 9 am every day, the excess drinking water without or mixed ENR-SLNs (corresponding $25 \mathrm{mg}$ ENR/L) was added and the remainder was weighted at 9 am of the next day. The daily water intake of each group was equal to the added water volume of the first day minus the remaining water volume of the next day.

\section{Evaluation of the granules}

\section{Granularity}

The granularity was tested by double sieve method according to the Chinese Veterinary Pharmacopoeia (2015). Briefly, $8.0 \mathrm{~g}$ of drugs were kept in a sieve with horizontal state and sifting by left and right for 3 minutes. The granules that cannot pass through the No 1 sieve with $2 \mathrm{~mm}$ pore diameter and powder that can pass through No 5 sieve with $0.2 \mathrm{~mm}$ pore diameter were weighed to calculate their proportions to the total granules.

\section{Drying loss}

ENR enteric-coated granules $(8.0 \mathrm{~g})$ were tiled in an open pan and placed in a drying oven with $40^{\circ} \mathrm{C}$. At fixed time point, the granules were weighed until the weight of granules was kept constantly. The weight loss of the samples on drying was calculated from the added amount minus the remainders. The assay was repeated in triplicate using different batch preparations.

\section{Stability}

The stability of ENR enteric granules was evaluated by influencing factor experiment including high temperature, high humidity, and strong light. The enteric granules were placed in a container and then placed in the environment of $60^{\circ} \mathrm{C}$ and $40^{\circ} \mathrm{C}$ (high temperature test), $25^{\circ} \mathrm{C}$ and $90 \% \pm 5 \%$ (humidity test), or 4,500 $\pm 500 \mathrm{l} \times$ (illumination) for 10 days, respectively. Samples were taken on the fifth and tenth day to assess the change of their appearance, drug content, granularity, and in vitro release performance.

\section{Pharmacokinetics}

Twelve healthy pigs before experiment were randomly divided into two groups (ENR soluble powder group and enteric-coated granules group) with six pigs in each group. ENR enteric granules (content: 10\%) and ENR soluble powder (Guangdong Wens, content: 5\%), respectively, resuspended or dissolved in $20 \mathrm{~mL} \mathrm{CMCC-Na}$ were administered to the pigs by intragastric administration with $2.5 \mathrm{mg} / \mathrm{kg}$ bw. Blood was collected from the anterior vena cava of the pigs, and blank plasma was collected prior to administration. After drug administration, the blood was collected at $0.25,0.5,0.75,1,2,4,6,8,12,24,36,48$, and 72 hours. Three milliliters of blood were collected at each time point, placed in a centrifuge tube containing heparin sodium, and centrifuged at 4,000 r/min to separate the plasma. The drug concentration in the plasma was detected by HPLC after pretreatment.

Pharmacokinetic parameters were calculated by WinNonlin software (version 6.4; Pharsight Corporation, Mountain View, CA, USA). Through observation the plasma concentration-time profile, the maximum plasma concentration $\left(C_{\max }\right)$ and time to reach $C_{\max }\left(T_{\max }\right)$ were obtained. The area under the plasma concentration-time curve $\left(\mathrm{AUC}_{0-\text { last }}\right)$ was determined using the log-linear trapezoidal rule.

\section{Quantitative measurement of ENR}

In order to quantitative measurement of ENR, $0.8 \mathrm{~mL}$ of plasma was measured into a $10 \mathrm{~mL}$ centrifuge tube containing $1.2 \mathrm{~mL}$ acetonitrile. The plasma and acetonitrile were thoroughly mixed under vortex for 2 minutes to precipitate protein and then centrifuged at $12,000 \mathrm{r} / \mathrm{min}$ for 20 minutes to take the supernatant. The supernatant was dried in nitrogen and reconstitute with $800 \mu \mathrm{L}$ mobile phase. The liquid was filtered through a $0.22 \mu \mathrm{m}$ filter and subjected to an HPLC (Waters Corp.) for detection. Chromatographic conditions were as follow: column: Agilent $\mathrm{SB} \mathrm{C}_{18}(250 \times 4.6 \mathrm{~mm} \times$ $5 \mu \mathrm{m})$; detection wavelength: $278 \mathrm{~nm}$; column temperature: $25^{\circ} \mathrm{C}$; mobile phase: $0.1 \%$ formic acid (phase A) and acetonitrile (phase B) with the ratio of 86:14; flow rate: $1.00 \mathrm{~mL} / \mathrm{min}$; and injection volume: $40 \mu \mathrm{L}$. The content of ENR in solution and plasma was determined using a standard curve. The HPLC method was validated in terms of linearity, accuracy, precision, and limit of detection and quantitation. ENR had a linear range from 0.1 to $1.8 \mu \mathrm{g} / \mathrm{mL}\left(R^{2}=0.9985\right)$. The limit of detection and quantitation were 0.025 and $0.08 \mu \mathrm{g} / \mathrm{mL}$, 
respectively. The relative $\mathrm{SD}$ of precision was $<2 \%$, and the recovery rates of three different added concentrations were $96.8 \%-103.3 \%$.

\section{Statistical analysis}

Data are presented as mean $\pm \mathrm{SD}$. Statistical significance was defined at $P$-value of 0.05 and 0.01 by the SPSS software (version 20, IBM).

\section{Results}

\section{Optimization of ENR-SLNs}

The maximum solubility of ENR in both melted octadecanoic acid and docosanoic acid is $33.33 \%$. Due to the cheap price, the octadecanoic acid was selected as lipid matrix. The optimal ratio of drug to lipid was 1:3. According to the analysis of orthogonal test, the size of ENR-SLNs was in the range of nanosize when the PVA was used as emulsifier. The PDI of sample 1 and 3 was $0.229 \pm 0.007$ and $0.155 \pm 0.030$, respectively while the other samples exceeded 0.5 (Table 2 ). The zeta potential of all the nine samples was negative. Considering the practicability of enteric granules, the LC was the most important to select the formulation. The factors influencing the LC were in the orders: emulsifier type $>$ concentration $>$ volume (Table 2 ). The final optimal aqueous phase is $10 \mathrm{~mL}$ of $3 \%$ PVA per $0.8 \mathrm{~g}$ ENR and $2.4 \mathrm{~g}$ octadecanoic acid.

\section{Physicochemical properties of ENR-SLNs}

As shown in Figure 2, scanning electron microscopy observation demonstrated that the ENR-SLNs were spherical in shape with relatively uniform distribution in bulk. The mean sizes, PDI, and zeta potential of the optimal SLNs were $308.5 \pm 6.3 \mathrm{~nm}, 0.352 \pm 0.015$, and $-22.3 \mathrm{mv}$, respectively. The LC and EE of the optimal SLNs were $15.73 \% \pm 0.31 \%$ and $68.78 \% \pm 1.35 \%$, respectively. Then the ENR-SLNs with these physicochemical properties were used to later researches.

\section{The effect of coating materials on in vitro release of granules}

In vitro release of ENR-SLNs and enteric granules in SIF and SGF is shown in Figures 3 and 4. In the SGF $(\mathrm{pH}=2)$, the ENR-SLNs and simple coating granules showed a closed release profiles but slightly slower release rate compared to native ENR. Note that, $96.56 \%$ of native ENR was swiftly dissolved in the simulated SGF within 1 hour, while about 95.62\% was released from the SLNs within 4 hours and $95.17 \%$ was released from the simple coating granules within 6 hours, suggesting that the sustained-release performance of ENR-SLNs and simple coating granules was not obvious. In vitro release of granules with different coating materials was significantly slower than those of the native ENR, ENRSLNs, and simple coating granules (Figure 3). The release velocity of enteric granules was decreased with the increase of the PRII content from $5 \%$ to $15 \%$. In order to achieve the slowest release in SGF, the $15 \%$ PRII was selected as the optimal coating material. When using the $10 \%$ ethyl cellulose, the release velocity of granules was the slowest compared to the other granules. Only half of the ENR was released from the granules within 36 hours.

In the SIF ( $\mathrm{pH}=8$ ), the ENR-SLNs showed a closed release profiles but slower release rate compared to native ENR (Figure 4). Note that, 97.15\% native ENR was dissolved in

Table 2 The optimization of emulsifier by orthogonal experiment

\begin{tabular}{|c|c|c|c|c|c|c|c|}
\hline Sample & Type & Concentration & Volume & LC (\%) & Size $(\mu \mathrm{m})$ & Zeta potential & PDI \\
\hline I & 1 & 3 & 2 & $14.74 \pm 0.59$ & $0.458 \pm 0.003$ & -20.8 & $0.229 \pm 0.007$ \\
\hline 2 & 3 & 3 & 3 & $12.49 \pm 0.45$ & $1.70 I \pm 0.030$ & -43.2 & $>0.5$ \\
\hline 3 & 1 & 2 & 3 & $15.66 \pm 0.25$ & $0.459 \pm 0.004$ & -24.2 & $0.155 \pm 0.030$ \\
\hline 4 & I & I & I & $|4.65 \pm 0.4|$ & $0.87 I \pm 0.050$ & -29.9 & $>0.5$ \\
\hline 5 & 3 & I & 2 & $12.46 \pm 0.28$ & $2.416 \pm 0.001$ & -33.1 & $>0.5$ \\
\hline 6 & 2 & I & 3 & $8.66 \pm 0.37$ & $2.097 \pm 0.002$ & -29.8 & $>0.5$ \\
\hline 7 & 2 & 3 & I & $13.34 \pm 0.45$ & $7.875 \pm 0.004$ & -28.5 & $>0.5$ \\
\hline 8 & 2 & 2 & 2 & $8.48 \pm 0.36$ & $5.566 \pm 0.004$ & -32.4 & $>0.5$ \\
\hline 9 & 3 & 2 & I & $11.78 \pm 0.32$ & $2.459 \pm 0.003$ & -23.2 & $>0.5$ \\
\hline KI & $14.99 \pm 0.65$ & $11.89 \pm 2.47$ & $13.23 \pm 1.20$ & & & & \\
\hline K2 & $10.16 \pm 2.29$ & $11.97 \pm 2.95$ & $11.89 \pm 2.62$ & & & & \\
\hline K3 & $12.24 \pm 0.48$ & $13.52 \pm 1.06$ & $12.27 \pm 2.89$ & & & & \\
\hline$R$ & 2.75 & 1.63 & 1.34 & & & & \\
\hline Optimum & $\mathrm{Al}$ & B3 & $\mathrm{Cl}$ & & & & \\
\hline
\end{tabular}

Notes: K1, K2, and K3 are the average of grade of LC for three levels in each factor; $\mathrm{R}$ is the different value between the max and mix of KI, K2, and K3 in each level. Abbreviations: LC, loading capacity; PDI, polydispersion index. 


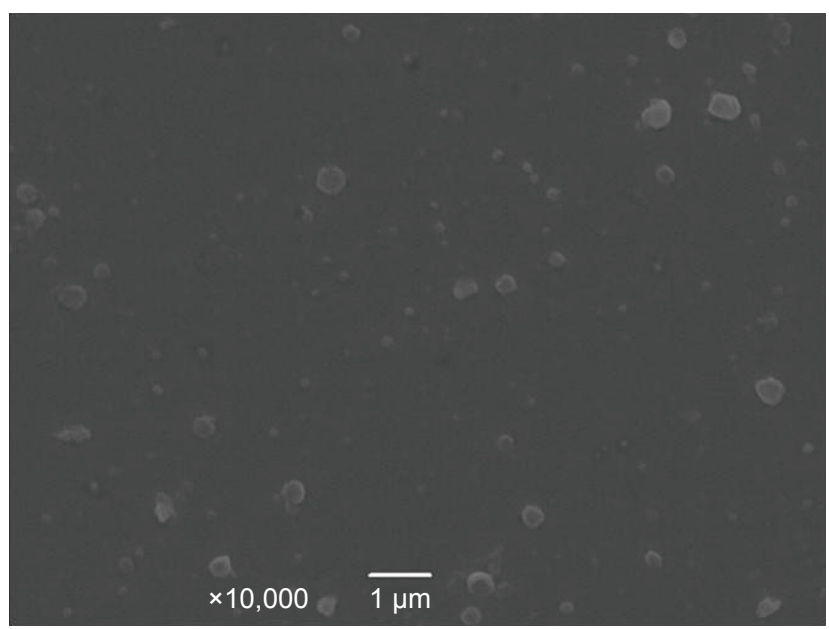

Figure 2 Scanning electron microscopy photographs of enrofloxacin-loaded SLNs. Abbreviation: SLNs, solid lipid nanoparticles.

the SIF within 4 hours, while the SLNs were released about 94.27\% within 8 hours, suggesting that ENR-SLNs held a certain sustained-release performance than native ENR. The enteric granules with $15 \%$ PRII as coating materials were slower than those of the native ENR and ENR-SLNs and significantly swifter than those in the $\mathrm{SGF}(\mathrm{pH}=2)$. These results demonstrated that the enteric granules, combined with SLNs with $15 \%$ PR coating had a positioning release in intestine.

\section{Palatability of different ENR formulations}

The normal daily feed intake and daily intake of feed medicated with different ENR formulations are shown in Table 3.
The daily feed intake of pigs in different groups was similar during the first 3 days before experiment, suggesting that the selected pigs were physiologically consistent. During the experiment, the daily feed intake of pigs in the ENR powder group ( $5 \mathrm{mg} / \mathrm{kg} \mathrm{bw}$ ) was sharply decreased from about $1.5 \mathrm{~kg} /$ day to below $0.2 \mathrm{~kg}$ /day. In contrast, the daily feed intake of pigs in the granules group (consists of $10 \%$ or $15 \%$ sucrose) ( $5 \mathrm{mg} / \mathrm{kg} \mathrm{bw}$ ) showed no significant difference compared to those of control groups and the normal daily feed intake. In the simple enteric coating granules $(5 \mathrm{mg} / \mathrm{kg} \mathrm{bw})$, the daily feed intake of pigs in the granules group was lower than those of control group and granules groups. The average of water intake of control group was $9.7 \pm 1.2 \mathrm{~L} /$ group/day, while the average daily water intake medicated with ENR-SLNs $(25 \mathrm{mg} / \mathrm{L})$ was decreased to $7.5 \pm 1.6 \mathrm{~L} /$ group/day. These results indicated that mere SLNs cannot completely overcome the palatability of ENR. The enteric granules combined SLNs with enteric-coating significantly improved the palatability of ENR and will help for the application of ENR in clinic.

\section{The properties of ENR enteric granules}

The optimal ENR enteric granules formulation was used; $15 \%$ PRII as coating materials and 10\% sucrose as flavoring agent. The ENR enteric granules were assessed following the Chinese Veterinary Pharmacopoeia (2015). The average total proportion of granules that pass through No 5 sieve and cannot pass through the No 1 sieve to the total granules was

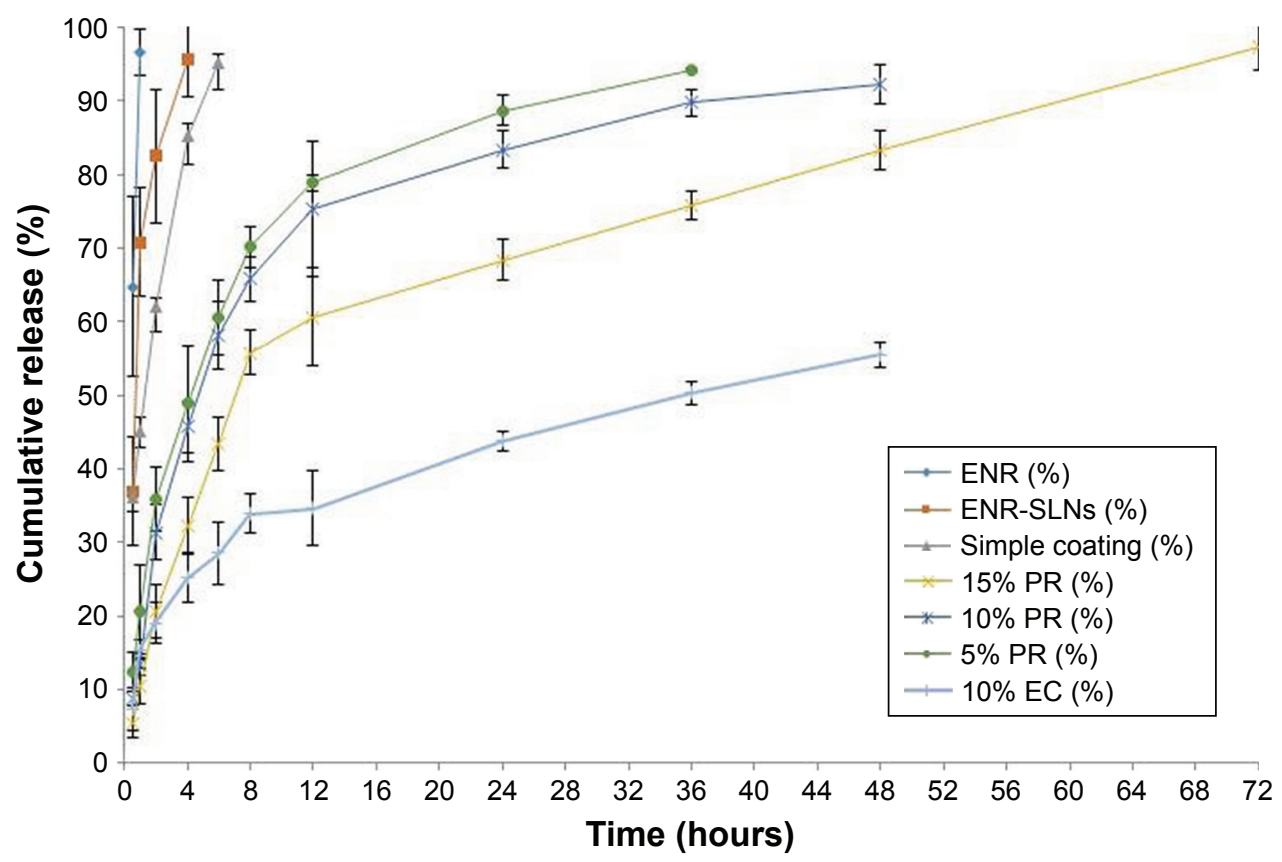

Figure 3 The accumulation release profiles of SLNs and granules in the simulated SGF $(p H=2)(n=3)$. 


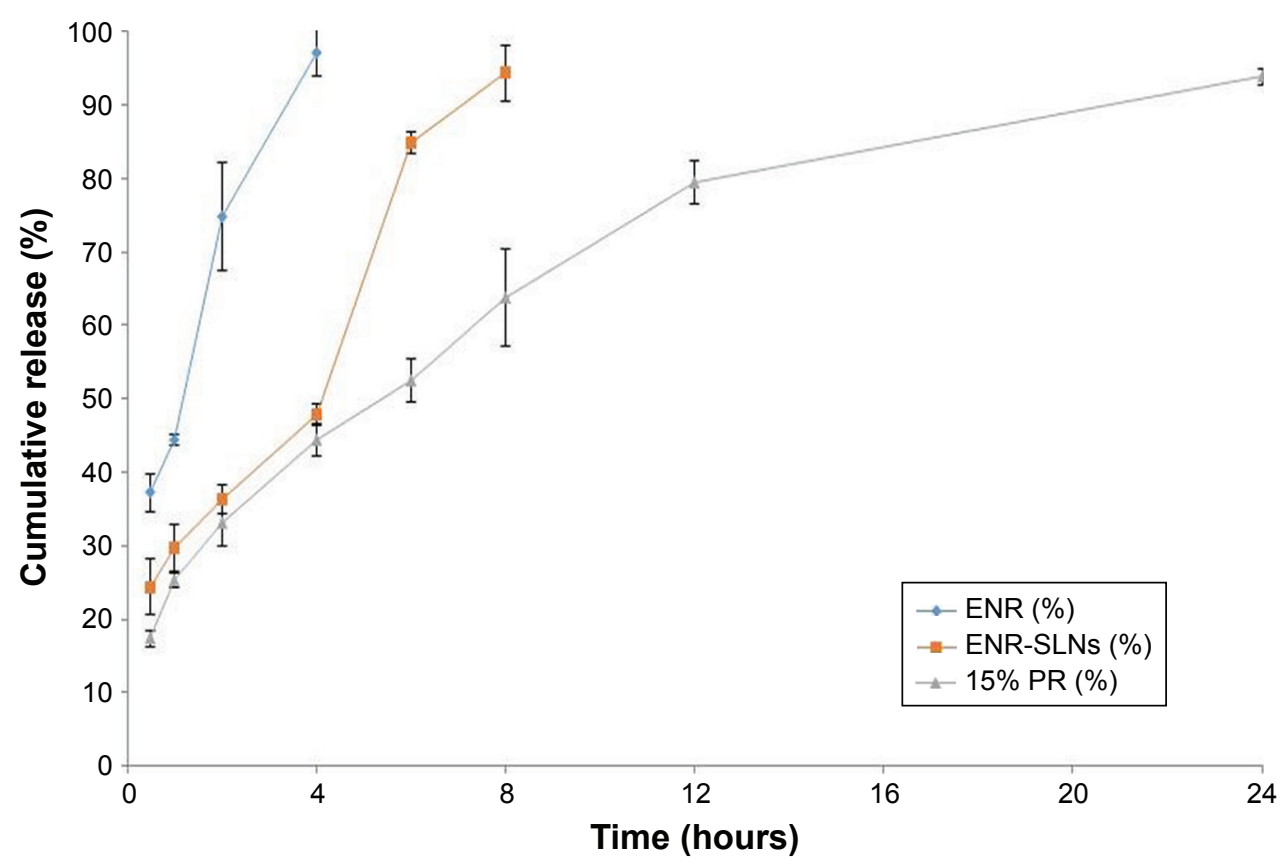

Figure 4 The accumulation release profiles of SLNs and granules in the simulated SIF $(p H=8)(n=3)$.

Abbreviations: ENR, enrofloxacin; ENR-SLNs, enrofloxacin-loaded SLNs; PR, polyacrylic resin; SIF, simulated intestine fluid; SLNs, solid lipid nanoparticles.

$7.60 \% \pm 0.12 \%$, which is less than the pharmacopeia requirement of $15 \%$. So the size of most ENR enteric granules (92.4\%) was $0.2-2 \mathrm{~mm}$. The average proportion of weight loss on drying is $1.90 \% \pm 0.10 \%$ and less than the pharmacopeia requirement of $2.0 \%$.

\section{Stability of granules}

The results of influencing factor experiment of granules including high temperature, high humidity, and strong light are shown in Table 4 . The appearance of enteric granules was white and not changed during the whole period of influencing factor experiment. In the strong light, only $4.89 \%$ and $6.0 \%$ of ENR in the granules was degraded after 5 and 10 days, respectively, while up to $15 \%$ and $20 \%$ of the native ENR was degraded after 5 and 10 days, respectively. These results demonstrated that the granules can improve the stability of ENR against high light. The ENR granules were stabile at high temperature of $40^{\circ} \mathrm{C}$ but slightly sensitive to the high temperature of $60^{\circ} \mathrm{C}$. In the high humidity, $4.82 \%$ and $7.0 \%$ of ENR in the granules was degraded after 5 and 10 days, respectively. The granularity of ENR enteric granules showed no obvious change. The amount and granularity of ENR enteric granules met the requirements of Chinese Veterinary Pharmacopeia (labeled amount: 90\%-110\%, unqualified granularity: $<15 \%$ ) after 10 -day influencing factor test. The release velocity of the ENR enteric granules under the condition of high temperature and high light for 5 and 10 days showed no obvious change while was slightly quicker under high humidity for 10 days (Figure 5). These results suggest that the enteric granules had good stability.

Table 3 The daily feed intake of pigs (mean $\pm S D, n=3$ )

\begin{tabular}{l|l|l|l|l|l|l|l|l|l|l}
\hline \multirow{2}{*}{ Groups } & \multicolumn{4}{l|}{ Before experiment (kg/group) } & \multicolumn{6}{l}{ During experiment (kg/group) } \\
\cline { 2 - 10 } & $\mathbf{I}$ day & $\mathbf{2}$ days & $\mathbf{3}$ days & $\overline{\mathbf{x}} \pm$ SD & I day & $\mathbf{2}$ days & $\mathbf{3}$ days & 4 days & $\mathbf{5}$ days & $\overline{\mathbf{x}} \pm$ SD \\
\hline Control & 1.55 & 1.60 & 1.42 & $1.52 \pm 0.09$ & 1.55 & 1.60 & 1.62 & 1.70 & 1.54 & $1.60 \pm 0.06$ \\
Enrofloxacin & 1.44 & 1.59 & 1.62 & $1.55 \pm 0.09$ & 0.11 & 0.19 & 0.15 & 0.17 & 0.12 & $0.15 \pm 0.03^{\mathrm{a}}$ \\
Simple coating & 1.56 & 1.57 & 1.58 & $1.57 \pm 0.01$ & 0.76 & 1.08 & 1.22 & 1.22 & 1.01 & $1.06 \pm 0.08^{\mathrm{a}, \mathrm{b}}$ \\
$10 \%$ Granules & 1.41 & 1.45 & 1.58 & $1.48 \pm 0.08$ & 1.15 & 1.22 & 1.56 & 1.46 & 1.74 & $1.43 \pm 0.24^{\mathrm{b}, \mathrm{c}}$ \\
$15 \%$ Granules & 1.60 & 1.54 & 1.58 & $1.57 \pm 0.03$ & 1.49 & 1.52 & 1.80 & 1.68 & 1.89 & $1.67 \pm 0.17^{\mathrm{b}, \mathrm{d}}$ \\
\hline
\end{tabular}

Notes: Control group was addition feed only; $10 \%$ granules was $10 \%$ sucrose included in granules; and $15 \%$ granules was $15 \%$ sucrose included in granules. aStatistical significance compared with control is $P<0.0$ l. bStatistical significance compared with feed mix ENR is $P<0.01$. 'Statistical significance compared with simple coating is $P<0.05$. 'Statistical significance compared with simple coating is $P<0.01$.

Abbreviation: ENR, enrofloxacin. 
Table 4 The influence factor test of granules (mean $\pm S D, n=3$ )

\begin{tabular}{l|l|l|l|l|l|l|l}
\hline Groups & Factors & \multicolumn{2}{l|}{ Content (\%) } & \multicolumn{2}{l}{ Unqualified granularity (\%) } \\
\cline { 3 - 7 } & & 0th day & 5th day & 10th day & 0th day & 5th day & 10th day \\
\hline Enrofloxacin & High light & 96.0 & $81.6 \pm 1.13$ & $76.8 \pm 2.12$ & $/$ & $/$ & $/$ \\
granules & High light & 10.05 & $9.56 \pm 2.55$ & $9.45 \pm 1.18$ & $7.56 \pm 0.13$ & $7.76 \pm 0.11$ & $7.73 \pm 0.14$ \\
& High temperature $60^{\circ} \mathrm{C}$ & 10.05 & $9.97 \pm 3.10$ & $9.23 \pm 3.56$ & $7.87 \pm 0.09$ & $7.73 \pm 0.15$ & $7.83 \pm 0.11$ \\
& High temperature $40^{\circ} \mathrm{C}$ & 10.05 & $9.86 \pm 2.31$ & $9.54 \pm 1.34$ & $7.33 \pm 0.20$ & $7.32 \pm 0.08$ & $7.02 \pm 0.18$ \\
& High humility & 10.05 & $9.57 \pm 1.69$ & $9.35 \pm 2.10$ & $6.89 \pm 0.15$ & $7.05 \pm 0.21$ & $7.23 \pm 0.16$ \\
\hline
\end{tabular}

\section{Pharmacokinetics}

After intragastric administration of the granules, the ENR in plasma was swiftly reached to the peak concentrations of $0.52 \pm 0.05 \mu \mathrm{g} / \mathrm{mL}$ at $3.33 \pm 1.03$ hours, then decreased slowly and sustained above $0.03 \mu \mathrm{g} / \mathrm{mL}$ for 72 hours. Comparatively, the soluble powder more quickly reached to the peak value of $0.60 \pm 0.12 \mu \mathrm{g} / \mathrm{mL}$ at $1.12 \pm 0.44$ hours, and then declined rapidly to $0.03 \mu \mathrm{g} / \mathrm{mL}$ at 24 hours after intragastric administration (Figure 6). The pharmacokinetic parameters are shown in Table 5. The $\mathrm{AUC}_{0 \text {-last }}, T_{1 / 2 \beta}$, and MRT of the granules were $11.24 \pm 3.33 \mu \mathrm{g} \mathrm{h} / \mathrm{mL}, 12.59 \pm 3.53$ hours, and $17.97 \pm 4.01$ hours, respectively, while these parameters for soluble powder were $4.26 \pm 0.85 \mu \mathrm{g} \mathrm{h} / \mathrm{mL}, 4.71 \pm 1.58$ hours

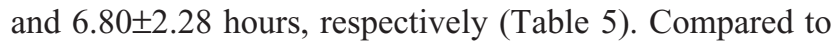
the soluble powder, the bioavailability, $T_{1 / 2 \beta}$, and MRT of the prepared ENR enteric granules were increased by 2.64-, 2.67-, and 2.64-folds, respectively. The results suggested that the prepared granules had sustained-release effect and improve the oral bioavailability of ENR.

\section{Discussion}

Coating technologies and nanoparticles are always used to produce the function preparations with ability of controlled ${ }^{23,24}$ and target release ${ }^{25}$ and to improve the palatability of the poor-tasting drugs. ${ }^{26}$ However, the single-coating granules, tablets, capsules, and other formulations will be chewed up by
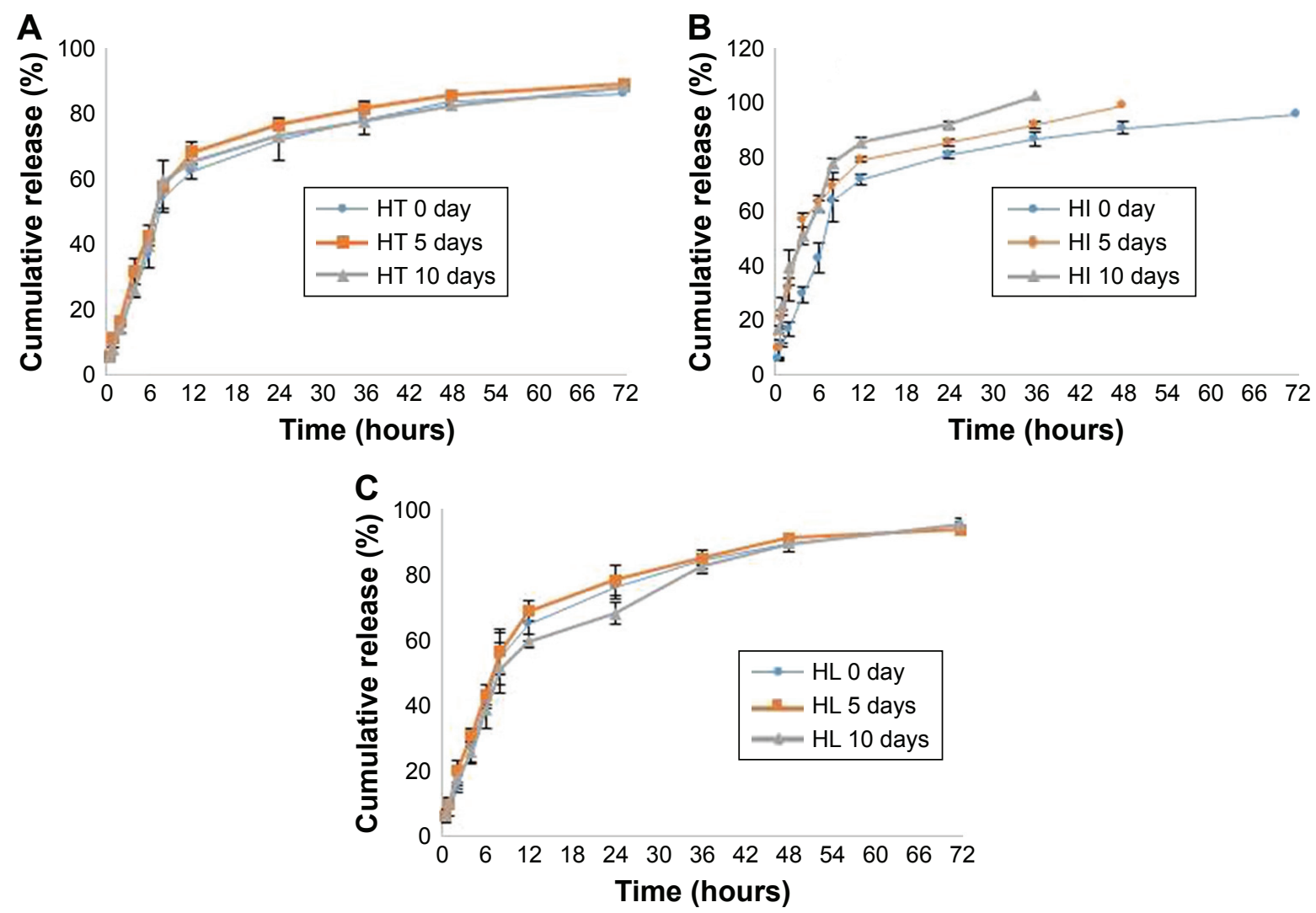

Figure 5 The influence of influencing factors experiments to release ability of enteric granules $(n=3)$.

Notes: (A) The influence of high temperature to release ability of enteric granules. (B) The influence of high humidity to release ability of enteric granules. (C) The influence of high light to release ability of enteric granules. $\mathrm{HT}$ : high temperature $\left(40^{\circ} \mathrm{C}\right)$; $\mathrm{HI}$ : high humidity $\left(25^{\circ} \mathrm{C}, 90 \% \pm 5 \%\right)$; $\mathrm{HL}$ : high light $(4,500 \pm 500 \mathrm{Ix})$. 


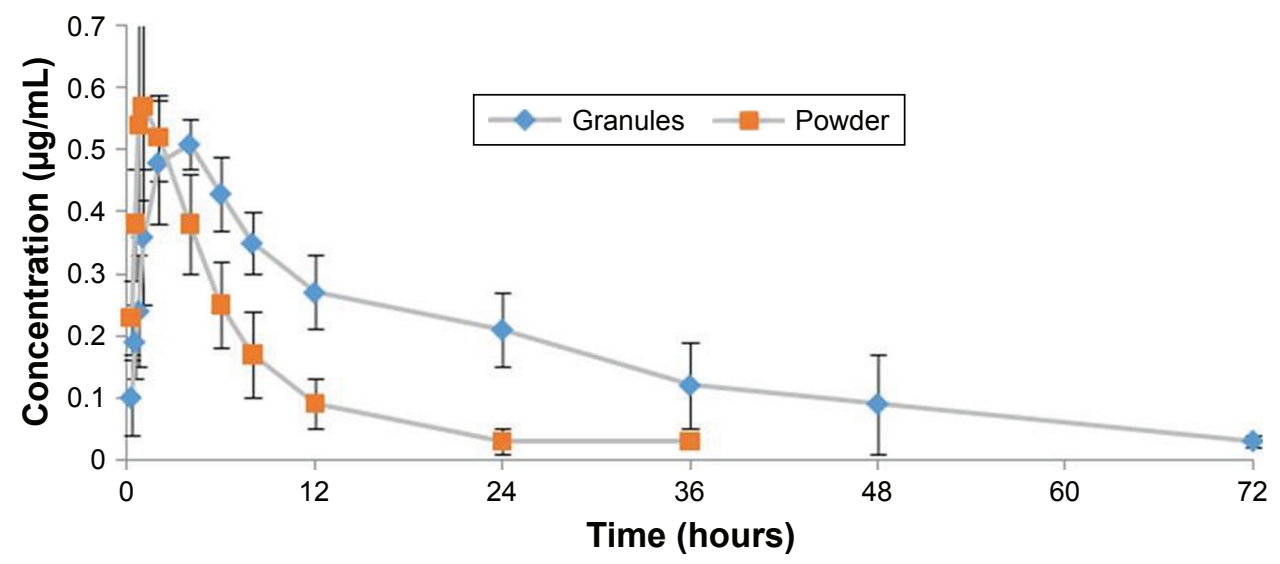

Figure 6 The plasma enrofloxacin concentration profiles - time of the prepared granules and reference formulation (soluble powder) in pigs ( $\mathrm{n}=6$ ). Notes: Granules: 10\% enrofloxacin enteric granules; powder: $5 \%$ enrofloxacin soluble powder.

animals. Thus, the coated drugs will be released and attached with the taste buds of animal mouth. This study also demonstrated that the daily feed intake of pig medicated with singlecoating granules was improved compared to those medicated with ENR powder but still less compared to the control group. These results suggested that the single-coating granules did not completely solve the bitterness of ENR. It is reported that lipid nanoparticles are also effective ways to improve the palatability of bitter-tasting drug since the drugs can be encapsulated and distributed into lipid materials. ${ }^{3}$ Meanwhile, SLNs are often used to improve the oral bioavailability of encapsulated drugs because of the adhesion of the nanosize of the particles. ${ }^{27}$ However, SLNs could not completely entrap the drug and often hold a burst release, and thus the nanoparticles may not entirely mask the bitter taste of encapsulated drug. Our study also showed that the daily water intake of pigs in the octadecanoic acid nanosuspension group was slightly lower than those of normal pigs. In order to overcome the problems of poor palatability and variable bioavailability of

Table 5 Pharmacokinetic parameters for ENR after oral administration of ENR enteric granules and ENR soluble powder in pigs (mean $\pm S D, n=6$ )

\begin{tabular}{l|l|l|l}
\hline Parameter & Unit & Enteric granules & Soluble powder \\
\hline $\mathrm{Vd}$ & $\mathrm{mL} / \mathrm{kg}$ & $3,671.07 \pm 768.5^{\mathrm{a}}$ & $5, \mathrm{II} 6.35 \pm \mathrm{I}, 47 \mathrm{I} .56$ \\
$\mathrm{MRT}$ & $\mathrm{h}$ & $17.97 \pm 4.0 \mathrm{I}^{\mathrm{b}}$ & $6.80 \pm 2.28$ \\
$\mathrm{AUC} \mathrm{C}_{0-\text { last }}$ & $\mu \mathrm{g} \mathrm{h} / \mathrm{mL}$ & $11.24 \pm 3.33^{\mathrm{c}}$ & $4.26 \pm 0.85$ \\
$T_{1 / 2 \beta}$ & $\mathrm{h}$ & $12.59 \pm 3.53^{\mathrm{d}}$ & $4.7 \mathrm{I} \pm \mathrm{I} .58$ \\
$\mathrm{CL}$ & $\mathrm{L} / \mathrm{h} / \mathrm{kg}$ & $240.19 \pm 62.76$ & $579.86 \pm 130.84$ \\
$T_{\max }$ & $\mathrm{h}$ & $3.33 \pm \mathrm{I} .03$ & $1.12 \pm 0.44$ \\
$C_{\max }$ & $\mu \mathrm{g} / \mathrm{mL}$ & $0.52 \pm 0.05$ & $0.60 \pm 0.12$ \\
$\mathrm{~F} \mathrm{( \% )}$ & $/$ & $263.85 \%$ & $/$ \\
\hline
\end{tabular}

Note: ${ }^{a, b, c, d}$ Statistical significance compared with soluble powder is $P<0.01$. Abbreviations: $\mathrm{AUC}_{0 \text {-last }}$, area under the curve; $\mathrm{CL}$, body clearance; $C_{\max }$, maximum plasma concentration; ENR, enrofloxacin; F, relative bioavailability; MRT, mean residence time; $T_{1 / 2 \beta}$, elimination half-life; $T_{\max }$, time to reach the $C_{\max }$.
ENR., the enteric coating granules were explored using the SLNs as inner core. The granules containing ENR-SLNs were prepared by wet granulation technology and followed by the coating using the polymer film-forming materials.

In the preparation of SLNs, the choice of lipid is crucial in the formulation design. Lipids must be selected based on their good ability to solubilize the encapsulated drug. Our previous work showed that the fatty acid has a high dissolution of ENR compared to the other melted lipids since ENR can be freely soluble in the acid solution. ${ }^{28}$ Considering the shortchain fatty acids with low melting point are inconvenient for storage and transportation, hexadecanoic acid, decanoic acid, and lauric acid were not within the scope of consideration. Therefore, the maximum solubility of ENR in melted longchain fatty acids of octadecanoic acid and docosanoic acid was determined in order to select the optimum encapsulated lipid matrix. The octadecanoic acid was finally selected due to the cheap price and satisfactory solubility. In order to obtain the higher LC for satisfying the practicability in clinic, the emulsifier was optimized by using orthogonal experimental design. According to the LC, size, and PDI, the SLN optimal formulation with $10 \mathrm{~mL} \mathrm{3 \%}$ PVA was selected for further characterizations. After formulation of the ENR-SLNs, different amounts of sucrose and starch were added into the nanosupension as flavoring and diluter agents according to the previous report, palatability, and cheap price.

In order to achieve the best palatability and sustained release, different coating materials were selected and evaluated in vitro release. In the SGF ( $\mathrm{pH}=2)$, the release of granules with different coating materials was significantly slower than those of the native ENR and ENR-SLNs. These results indicated that the granules with different amount of PRII were relatively stable in the gastric juice. The sustained release of 
granules with 5\% and 10\% PRII showed no significant difference while the granules with 15\% PRII showed the slowest release in SGF. Therefore, 15\% PRII content was used in the optimum formulation. When using $10 \%$ ethyl cellulose as coating materials, the release velocity of granules was the slowest compared to the granules with PRII as coating materials. However, ethyl cellulose is a kind of water-insoluble material, and its solubility is irrelevant with $\mathrm{pH} .{ }^{29}$ According to the solubility of ENR, the granules with $10 \%$ ethyl cellulose will also release very slowly in the simulated SIF $(\mathrm{pH}=8)$. Considering the gastrointestinal emptying time of pigs ( $\leq 50$ hours) ${ }^{30}$ the granules were formulated with $15 \%$ PRII but not using 10\% ethyl cellulose. In addition, ENR is a kind of concentration-dependent antibiotic; the effective exertion of antibacterial activity needs high $C_{\max }$ level. The release of granules with $15 \%$ PRII was slower than those of the native ENR and ENR-SLNs in the SGF ( $\mathrm{pH}=2)$ but swifter than itself in the $\mathrm{SIF}(\mathrm{pH}=8)$. The ENR might be released completely within the gastrointestinal emptying time of pigs and can keep higher $C_{\max }$ than coating by ethyl cellulose. These results demonstrated the granules combining SLNs with $15 \%$ PRII coating had a positioning release in intestine and decreased stomach irritating. Although ethyl cellulose was not used as coating material in our research, we proved that ethyl cellulose could be used to prepare excellent sustain release preparation by coating time-dependent antibiotics (ie, macrolides). The release of ENR from the inner SLN might be due to the diffusion combined with erosion. ${ }^{31-33}$ The ENR-SLNs released very fast in the SGF $(\mathrm{pH}=2)$ due to the highest solubility in the acid condition. The burst release might be the swift dissolution of free and nonentrapped drug and absorbed ENR on surface. For enteric granules containing SLNs, the main mechanism of drug release might be diffusion from the semipermeable membrane of coating of PRII due to the insoluble properties of outer PRII in SGF. Due to the double obstruction, the rate of the enteric granules was significantly slower than the SLNs. In SIF, the outer PRII coating of the granules was quickly dissolved, which leads to the disintegration of the granules. The release velocity of the granules in SIF mainly depended on the rate of granules disintegration and the drug diffusion from SLNs. Therefore, the ENR in granules was released quickly in SIF than in the SGF.

The palatability of the granules coated with $15 \%$ PRII was evaluated. The daily feed intake of pigs in the granules combined with SLNs groups (consists of $10 \%$ or $15 \%$ sucrose) $(5 \mathrm{mg} / \mathrm{kg} \mathrm{bw})$ was not statistically significantly different $(P<0.01)$ compared to those of control groups and the normal daily feed intake and higher than those of ENR powder group and simple enteric coating granules group. In order to decrease the price of the formulation, $10 \%$ sucrose was used as flavoring agents. These results indicated that the enteric granules, which combined SLNs with enteric-coating significantly, improved the palatability of ENR and will help for the application of ENR in clinic.

The granularity and weight loss on drying of the ENR granules met the requirements of the Chinese Veterinary Pharmacopoeia (2015). The stability of the prepared enteric granules under high temperature, high humidity, and strong light was shown to be good. Furthermore, the granules could significantly improve the stability of ENR in the strong light. This might be due to the lucifugal effect of lipid and coating material. The appearance, drug content, and granularity range of the enteric granules showed no obvious changes under the high temperature and humidity and strong light. This result suggested that the prepared enteric granules have a good stability. The core materials of the enteric granules also include the SLN, sucrose, and starch. Therefore, it is not possible to merely investigate the change of the inner SLNs. The in vitro release change of the granules under the high temperature and humidity and strong light was compared to the release of the initial preparation to indirectly evaluate the stability of the inner SLNs. The in vitro release profiles of enteric granules showed no change except the condition of high humidity for 10 days. These suggest that the enteric granules had good stability under high temperature and light while slightly sensitive to high humidity. Therefore, good moisture-proof measures were needed in storage and transportation of ENR enteric granules. The good stability of the enteric granules might be due to the excellent stability of inner nanoparticles and the coating of PRII. It is reported by Wang et al that the octadecanoic acid nanosuspension showed an excellent stability at room temperature for 9 months with no changes of particle size and zeta potential and slightly decrease of LC. ${ }^{34}$ It is well known that the stability of nanoparticles in solid powder sates is better than those in liquid. The good stability of ENR-SLNs within enteric granules is predictable. Therefore, the stability of inner ENR-loaded octadecanoic acid SLNs was not separately studied in this study.

After intragastric administration, the prepared enteric granules had sustained-release effect with improved bioavailability. Compared to soluble powder, the bioavailability, $T_{1 / 2 \beta}$, and MRT of the prepared ENR enteric granules were increased by 2.64-, 2.67-, and 2.64-folds, respectively. The improved bioavailability, controlled release, and palatability 


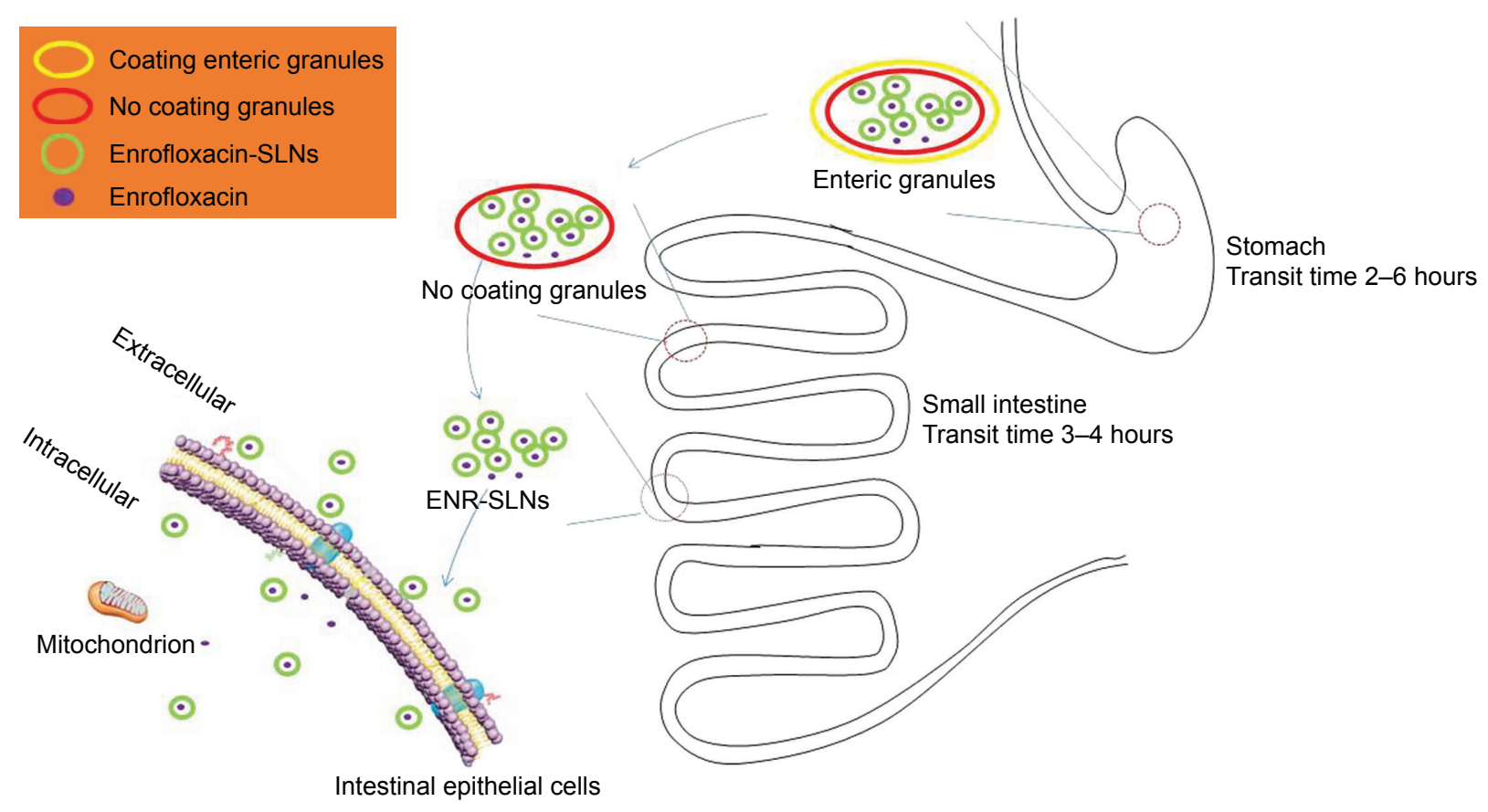

Figure 7 The transfer process of enrofloxacin granules in gastrointestinal tract of pigs. Abbreviations: ENR-SLNs, enrofloxacin-loaded SLNs; SLNs, solid lipid nanoparticles.

of the enteric granules might be closely related with the gastrointestinal transit (Figure 7). When the granules were taken by pig, they will taste good due to the isolation of ENR and taste bud in mouth by octadecanoic acid encapsulation and PRII coating. When the granules subsequently reach stomach, the inner ENR of the enteric granules will be less released within the time of gastric empty (2-6 hours) and will show little stimulation to the gastric mucosa. The increased bioavailability of the granules lessens the amount of ENR metabolized in stomach fluid resulting in more ENR reaching the intestine, . The granules begin to disintegrate due to the dissolution of outer layer PRII when they reach the small intestine whose $\mathrm{pH}$ is close to neutral by the gastric emptying. The inter ENR-SLNs were released following the granules disintegration. The granules showed higher oral bioavailability because of the high adhesion and permeability of ENR-SLNs due to the nanosize and large surface area.

In our previous work, we proved that ENR-SLNs showed a good transmembrane transport and thus produced higher intracellular accumulation and stronger antibacterial activity as well as longer antibacterial time against intracellular Salmonella spp in vitro. ${ }^{21}$ The pharmacokinetics indicated that enteric granules have the similar $C_{\max }$ compared with ENR soluble powder but longer maintenance time of blood concentration. The similar $C_{\max }$ and enhanced absorption of the enteric granules and enhanced antibacterial activity of inner nanoparticles against intracellular bacterial will not easily induce the resistance and will be helpful for the treatment of intestinal infections by intracellular bacterial such as Salmonella and Intracellular Lawson bacteria. The pharmacodynamics and the effect of the enteric granules on the intestinal pathogens will be studied to evaluate the possible application in clinic in the future.

\section{Conclusion}

The optimum ENR enteric granules were formulated by the selection of lipid materials, emulsifiers, coating material, and other accessories by orthogonal test or single factor screening. The ENR enteric granules combining SLNs with enteric coating significantly improved the stability, palatability, controlled release, and oral bioavailability of ENR. The combination of SLNs and enteric coating will be a potential measure to increase the stability, palatability, sustained release, and bioavailability of other drugs.

\section{Acknowledgment}

This work was supported by the National Key Research and Development Program of China (2017YFD0501402) and the National Natural Science Foundation of China (grant no 31772797$)$.

\section{Disclosure}

The authors report no conflicts of interest in this work. 


\section{References}

1. Ribeiro C, Lopes SC, Gameiro P. New insights into the translocation route of enrofloxacin and its metalloantibiotics. J Membr Biol. 2011; 241(3):117-125.

2. Yang F, Kang J, Yang F, Zhao Z, Kong T, Zeng Z. Preparation and evaluation of enrofloxacin microspheres and tissue distribution in rats. J Vet Sci. 2015;16(2):157-164.

3. Liu M, Yin D, Fu H, et al. Double-coated enrofloxacin microparticles with chitosan and alginate: preparation, characterization and tastemasking effect study. Carbohydr Polym. 2017;170:247-253.

4. Chun MK, Choi HK. Preparation and characterization of enrofloxacin/carbopol complex in aqueous solution. Arch Pharm Res. 2004; 27(6):670-675.

5. Zj L, Dong JN, Zeng BG. The new research progress of animals' physiological taste and application of sweetening agent. Feed Industry. 2005;26(20):1-4.

6. Mao C, Xie X, Liu X, et al. The controlled drug release by $\mathrm{pH}$-sensitive molecularly imprinted nanospheres for enhanced antibacterial activity. Mater Sci Eng C Mater Biol Appl. 2017;77:84-91.

7. Xue Y, Xia X, Yu B, et al. A green and facile method for the preparation of a $\mathrm{pH}$-responsive alginate nanogel for subcellular delivery of doxorubicin. RSC Adv. 2015;5(90):73416-73423.

8. Lizondo M, Pons M, Gallardo M, Estelrich J. Physicochemical properties of enrofloxacin. J Pharm Biomed Anal. 1997;15(12):1845-1849.

9. Zamanpour G, Mehrabani-Zeinabad A. An experimental study on bioremediation and photolysis of enrofloxacin. Water Sci Technol. 2014;70(5):932-938.

10. Xie S, Tao Y, Pan Y, et al. Biodegradable nanoparticles for intracellular delivery of antimicrobial agents. J Control Release. 2014;187:101-117.

11. Guo S, Pham K, Li D, Penzak SR, Dong X. Novel in situ self-assembly nanoparticles for formulating a poorly water-soluble drug in oral solid granules, improving stability, palatability, and bioavailability. Int $J$ Nanomedicine. 2016;11:1451-1460.

12. Shazly GA, Alshehri S, Ibrahim MA, et al. Development of domperidone solid lipid nanoparticles: in vitro and in vivo characterization. AAPS PharmSciTech. 2018;19(4):1712-1719.

13. Wang XF, Zhang SL, Zhu LY, et al. Enhancement of antibacterial activity of tilmicosin against Staphylococcus aureus by solid lipid nanoparticles in vitro and in vivo. Vet J. 2012;191(1):115-120.

14. Wang F, Cao J, Hao J, Liu K. Pharmacokinetics, tissue distribution and relative bioavailability of geniposide-solid lipid nanoparticles following oral administration. J Microencapsul. 2014;31(4):382-389.

15. Felton LA, Porter SC. An update on pharmaceutical film coating for drug delivery. Expert Opin Drug Deliv. 2013;10(4):421-435.

16. Sohi H, Sultana Y, Khar RK. Taste masking technologies in oral pharmaceuticals: recent developments and approaches. Drug Dev Ind Pharm. 2004;30(5):429-448.

17. Choi HG, Kim CK. Application of dry elixir system to oriental traditional medicine: taste masking of peonjahwan by coated dry elixir. Arch Pharm Res. 2000;23(1):66-71.
18. National Center for Biotechnology Information. PubChem Database; Patent=EP0458751, https://pubchem.ncbi.nlm.nih.gov/patent/ EP0458751. Accessed March 22, 2018.

19. Alsulays BB, Kulkarni V, Alshehri SM, et al. Preparation and evaluation of enteric coated tablets of hot-melt extruded lansoprazole. Drug Dev Ind Pharm. 2017;43(5):789-796.

20. Chen Z, Lu Y, Qi J, Wu W. Enhanced dissolution, stability and physicochemical characterization of ATRA/2-hydroxypropyl- $\beta$-cyclodextrin inclusion complex pellets prepared by fluid-bed coating technique. Pharm Dev Technol. 2013;18(1):130-136.

21. Xie S, Yang F, Tao Y, et al. Enhanced intracellular delivery and antibacterial efficacy of enrofloxacin-loaded docosanoic acid solid lipid nanoparticles against intracellular Salmonella. Sci Rep. 2017;7(1):4110.

22. Chinese Veterinary Pharmacopoeia. 2015 [S].

23. Nakamura S, Sakamoto T, Ito T, Kabasawa K, Yuasa H. Preparation of controlled-release fine particles using a dry coating method. AAPS PharmSciTech. 2016;17(6):1393-1403.

24. Wang S. Conjugated polymer nanoparticles appending photo-responsive units for controlled drug delivery, release and imaging. Angew Chem Int Ed Engl. 2018;57(40):13114-13119.

25. Maity S, Sa B. Compression-coated tablet for colon targeting: impact of coating and core materials on drug release. AAPS PharmSciTech. 2016; 17(2):504-515.

26. El Edelbi R, Eksborg S, Lindemalm S. In situ coating makes it easier for children to swallow and tolerate tablets and capsules. Acta Paediatr. 2015;104(9):956-961.

27. Fq L, Yan C, Bi J. A novel spray-dried nanoparticles-in-microparticles system for formulating scopolamine hydrobromide into orally disintegrating tablets. Int J Nanomedicine. 2011;6:897-904.

28. Xie S, Zhu L, Dong Z, et al. Preparation, characterization and pharmacokinetics of enrofloxacin-loaded solid lipid nanoparticles: influences of fatty acids. Colloids Surf B Biointerfaces. 2011;83(2):382-387.

29. Marucci M, Andersson H, Hjärtstam J, et al. New insights on how to adjust the release profile from coated pellets by varying the molecular weight of ethyl cellulose in the coating film. Int J Pharm. 2013;458(1): 218-223.

30. Davis SS, Illum L, Hinchcliffe M. Gastrointestinal transit of dosage forms in the pig. J Pharm Pharmacol. 2001;53(1):33-39.

31. Zur Mühlen A, Schwarz C, Mehnert W. Solid lipid nanoparticles (SLN) for controlled drug delivery-drug release and release mechanism. Eur J Pharm Biopharm. 1998;45(2):149-155.

32. Müller RH, Mäder K, Gohla S. Solid lipid nanoparticles (SLN) for controlled drug delivery - a review of the state of the art. Eur J Pharm Biopharm. 2000;50(1):161-177.

33. Sutch JC, Ross AC, Köckenberger W, et al. Investigating the coatingdependent release mechanism of a pulsatile capsule using NMR microscopy. J Control Release. 2003;92(3):341-347.

34. Wang Y, Zhu L, Dong Z, et al. Preparation and stability study of norfloxacin-loaded solid lipid nanoparticle suspensions. Colloids Surf B Biointerfaces. 2012;98:105-111.
International Journal of Nanomedicine

\section{Publish your work in this journal}

The International Journal of Nanomedicine is an international, peerreviewed journal focusing on the application of nanotechnology in diagnostics, therapeutics, and drug delivery systems throughout the biomedical field. This journal is indexed on PubMed Central, MedLine, CAS, SciSearch $\AA$, Current Contents ${ }^{\circledR} /$ Clinical Medicine,

\section{Dovepress}

Journal Citation Reports/Science Edition, EMBase, Scopus and the Elsevier Bibliographic databases. The manuscript management system is completely online and includes a very quick and fair peer-review system, which is all easy to use. Visit http://www.dovepress.com/ testimonials.php to read real quotes from published authors. 